\title{
Lacrimal surgery: proposal of a new surgical classification of endoscopic dacryocistohinostomy
}

\begin{abstract}
Endoscopic dacryocystorhinostomy (e-DCR) is a well-established surgical procedure with results that are equivalent to the classic open approach. Although the aim of surgery is to create a direct communication between the lacrimal sac and the lateral wall of the nose by drilling out the lacrimal bone, different endoscopic surgical techniques to the lacrimal sac are possible. However nowadays we do not have a surgical procedure classification system. In an effort to enhance scientific communication, we would like to propose a surgical classification system based on the different insertion modalities of the uncinate process (UP) on the lacrimal bone and on the anatomical variations that may eventually require additional surgical steps.
\end{abstract}

Volume 8 Issue 2 - 2017

\author{
Luca Malvezzi,' Armando DeVirgilio,' \\ Giovanni Colombo,' Giovanni Cugini,' \\ Stefano Miceli,' 'Vanessa Piera Rossi,', Susanna \\ Di Pietro,' Eleonora M Minerva, ${ }^{2}$ Nicolo \\ Stomeo ${ }^{2}$ \\ 'Department of Otorhinolaringology Humanitas Clinical and \\ research Center Italy \\ Department of Biomedical Sciences Humanitas University, Italy
}

Correspondence: Luca Malvezzi Department of

Otorhinolaringology Humanitas Clinical and research Center

Rozzano Milan, Italy, Email luca.malvezzi@humanitas.it

Received: July II, 2017 | Published: August 2, 2017

\section{Introduction}

Dacryocystorhinostomy (DCR) is the treatment of choice for primary nasolacrimal obstruction, responsible for chronic epiphora and dacryocystitis, which is the infection of the lacrimal sac. The surgical procedure was born and proposed by Toti in $1904^{1}$ as an open surgical technique (ex-DCR), which is still the preferred route by the majority of the ophthalmologists. ${ }^{2-4}$

In the following year's authors as Caldwell, ${ }^{5}$ West $^{6}$ and Mosher ${ }^{7}$ proposed the intranasal access to the lacrimal sac. However it was just in the last two decades of the past century that the endoscopic approach gained progressive interest, thanks to the exponential affirmation of the modern endoscopic technique. Nowadays the success rate of the two procedures, ex-DCR and endoscopic DCR (e-DCR), are considered equivalent. ${ }^{8-10}$

In 1989 McDought and Miering described the first endoscopic approach to the lacrimal sac. ${ }^{11}$ The progressive development of specialized endoscopic instruments in the ENT field allowed the proposal of different surgical approaches that we can find in literature. Few examples are the possible realization of a mucosal flap on the lateral nasal wall in order to expose the bone, successively to be drilled to realize the ostomy with the lacrimal sac; ${ }^{12}$ the utilization of different types of laser in the ostomy realization; ${ }^{13,14}$ the possibility not to use a canalicular silicon intubation tube ${ }^{15}$ mitomycin $\mathrm{C}$ to favor the optimal healing of the wound. ${ }^{16}$

However, the greatest difference in the surgical approach is represented by the different site of canalization with the lacrimal sac. In fact, the first option is to open the lacrimal sac through the burring of the frontal process of the maxillary bone. In that case the surgeon is forced to remove a thick portion of bone increasing the probability of an impaired healing, in particular by using the burr that exposes the region to a thermal insult.

The second option is to plan the surgical approach according to the anatomical relationships between the uncinate process (UP) and the lacrimal bone. According to our experience, this second approach is more conservative, since it is based on the pre-operative evaluation of the paranasal sinuses $\mathrm{CT}$ scan, in perfect agreement with the principle of endoscopic sinus surgery. ${ }^{17}$

In this paper we present a selected number of patients that underwent e-DCR proposing a surgical classification based on two key points: the different insertion the UP on the lacrimal bone and the anatomical variability of the surrounding anterior ethmoidal structures.

\section{Endoscopic surgical approach and anatomical considerations}

Surgical planning is based on preoperative CT scan evaluation, with a particular attention to the anatomical relationships between UP and lacrimal process and the anatomical variances of the anterior ethmoid bone. According to its articulation the UP can be classified as retro-lacrimal, lacrimal or pre-lacrimal. In the retro-lacrimal position, the UP does not hide the lacrimal sac projection on the lateral nasal wall. In the lacrimal position, the lacrimal sac projection is partially hidden, while in the pre-lacrimal position the UP hides completely the projection of the lacrimal sac from the endoscopic view. The anterior ethmoid anatomical variants, which most frequently influence the access to the lacrimal sac, are represented by UP pneumatization, UP retourning, agger nasi cell pneumatization and concha bullosa.

During the surgical procedure the use of a rigid light probe inserted in the inferior lacrimal punctum and canaliculus allows identifying the projection of the lacrimal sac on the lateral nasal wall (Figure 1). In fact, the site of major illumination helps the operator in defining where the bone is thinnest in the lacrimal fossa. This technique further confirms the relation between UP and lacrimal bone, already evaluated with CT. As shown in Figure 2, the prelacrimal UP hides the lacrimal bone making its removal necessary in order to realize the ostomy with the lacrimal sac.

\section{Classification proposal}

E-DCR Type 1(UP in retro-lacrimal position) (Figure 3a): in this case the transillumination with the light probe shows the projection of the lacrimal sac on the lateral nasal wall in front of the UP. The 
lacrimal bone is skeletonized with a diamond burr, without creating a mucosal flap to expose it, and then the lacrimal sac is identified and marsupialized.

E-DCR Type 2 (UP in lacrimal position) (Figure 3b): the portion of the UP that inserts on the lacrimal bone is carefully sectioned (superior uncinotomy). The lacrimal bone is exposed and drilled. Finally, the lacrimal sac is exposed and marsupialized.

E-DCR Type 3 (UP in pre-lacrimal position) (Figure 3c): inferior uncinotomy is required to find the natural ostium of the maxillary sinus. Then, via a gentle dissection, the vertical portion of the UP is removed. In this way, the lacrimal bone is exposed and drilled under light guidance. Finally, the lacrimal sac is marsupialized. After any of the described procedures, with the opening of the lacrimal sac, the lacrimal system is irrigated with antibiotic solution and then a bicanalicular silicone intubation is performed.

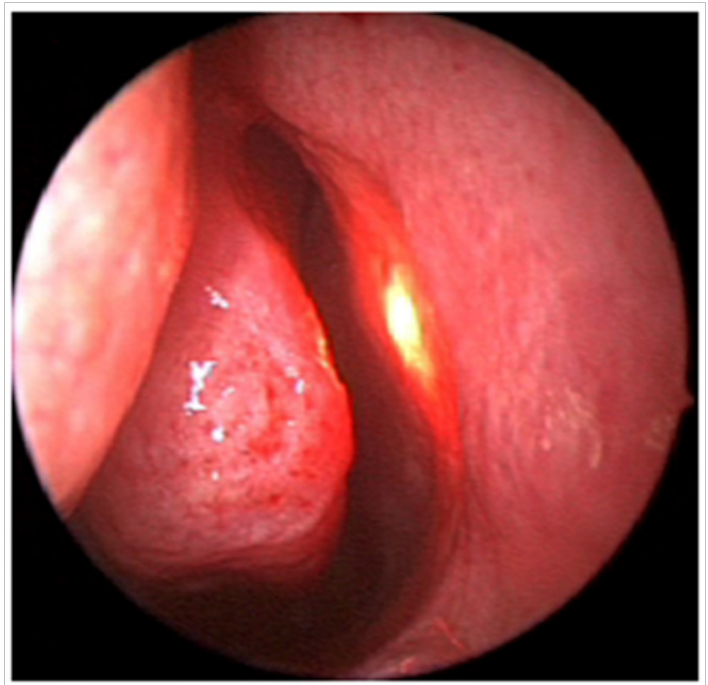

Figure I Left nostril - transillumination of the lacrimal sac.

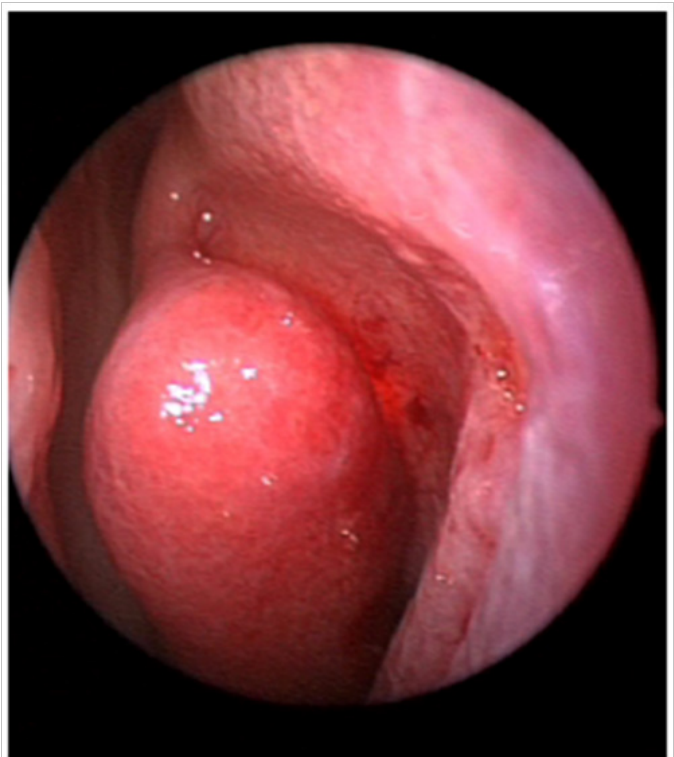

Figure 2 Left nostril - transillumination of the lacrimal sac impeded by prelacrimal insertion of UP.

Suffixes: we propose the use of the suffixes 'UPp', 'rUP', 'anp', 'cb', 's' in case of surgical anatomical variations whose correction is required prior to approach the lacrimal sac. "UPp" identifies the UP pneumatization, "UPr" identifies the returning UP, "anp" identifies the agger nasi pneumatization, "cb" identifies the pneumatization of the middle turbinate (concha bullosa). When septoplasty is required "s" suffix is added.

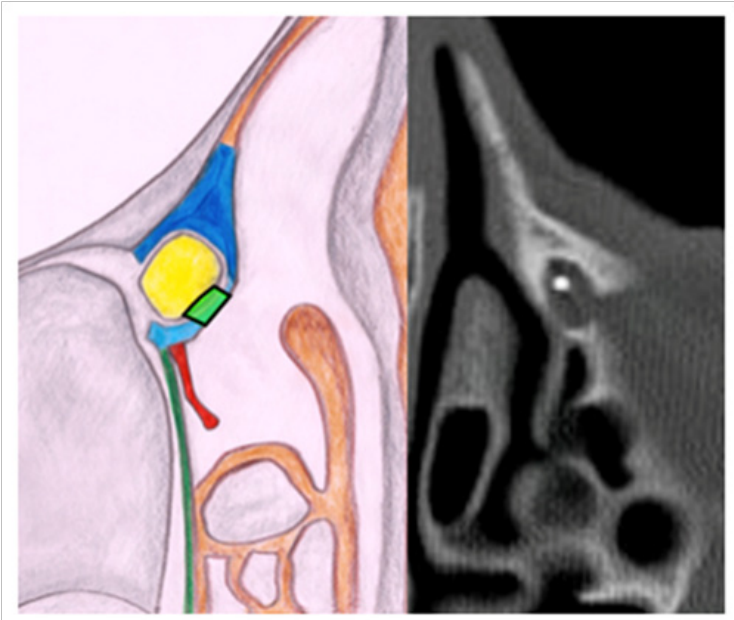

Figure 3a UP in retro-lacrimal position.

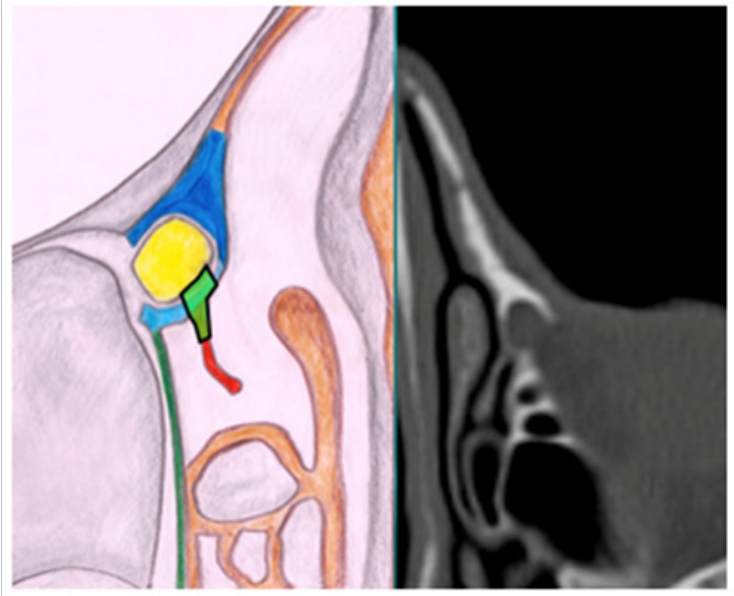

Figure $\mathbf{3 b}$ UP in lacrimal position.

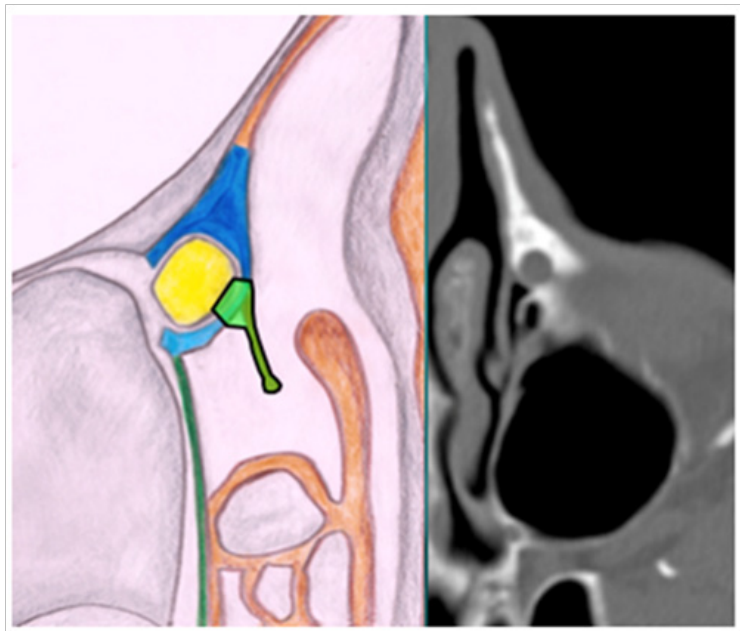

Figure 3c UP in lacrimal position. 


\section{Materials and methods}

Over the last ten years at Humanitas Clinical and Research Hospital, about 400 e-DCR surgery have been performed. After the application of exclusion criteria we included 150 patients (117 females, male 33) that underwent single-sided surgery between 2007 and 2014. Surgical approach was planned according to the insertion of the UP on the lacrimal bone.

Exclusion criteria were: follow-up shorter than two years, previous ex-DCR sinus surgery or recurrent epiphora after DCR done in other hospitals, concomitant granulocytic disease such as Wegener granulomatosis, allergic rhinitis, rhinosinusitis with or without nasal polyposis.

The 150 surgical procedures were performed by the same surgical team consisting of an otolaryngologist (LM, first author) and an ophthalmologist (ADM).

All the patients underwent pre-operatory ophthalmological examination in order to confirm the post-saccal stenosis, ENT examination and CT scan with inoculation of contrast media in the lacrimal duct. The preoperative $\mathrm{CT}$ was fundamental in order to evaluate the different insertion of the UP on the lacrimal bone and the anatomical variability of the surrounding anterior ethmoidal structures. The inoculation of contrast media is a key element in confirming the post saccal stenosis. In 32 cases $(21 \%)$ the UP was in a retrolacrimal position, in 9 cases $(6 \%)$ in lacrimal position and in 109 cases $(73 \%)$ in prelacrimal position. In 36 cases $(24 \%)$ a concha bullosa was evidenced and treated through the endoscopic removal of its lateral aspect. Anatomical variants were corrected in 69 patients $(46 \%)$. All the procedures were successfully completed in all patients without complications.

All the patients had a follow up visit on $7^{\text {th }}, 21^{\text {st }}$ post-operative day, then every 3 months during the first postoperative year; thereafter, once a year. During the first 3 weeks after surgery patients were suggested to perform nasal washings with hyaluronic acid twice a day to improve healing of the surgical site.

\section{Discussion}

E-DCR is now a well-established surgical procedure with results that can be superimposed to the ex-DCR. The optimum surgical result is achieved by ensuring long-term maintenance of the ostomy with the perforated lacrimal sac. According to our experience this is achieved by the careful dissection of the anatomical structures of the lateral nasal wall and the realization of a sufficiently wide ostomy with the lacrimal sac. These results are efficiently obtained using an endoscopic approach, as it is widely documented in contemporary literature. Post-operative wound care demonstrated to be fundamental as well. Hyaluronic acid inhalation proved effective in favoring scar healing. ${ }^{18}$

Although the endoscopic access to the lacrimal sac can be obtained via different sites, i.e. through the frontal process of the maxillary bone, it is widely demonstrated that the preferential approach is the least traumatic one. While the thickness of the frontal process of the maxillary bone imposes a traumatic removal, the thinner lacrimal bone is easily skeletonized with the burr. In this setting, the preoperative evaluation through a CT scan is crucial, since it allows the identification of the preferential surgical access site. Indeed, the anatomical relations between UP and lacrimal bone and also other possible anatomical variations of the anterior ethmoid bone deserve a consideration in surgical planning.
In our opinion the conventional use of a simple classification could optimize communication between surgeons and could facilitate endoscopic evaluations during follow-up. Furthermore, understanding which anatomical structures have been manipulated and eventually sacrificed can help the ENT specialist in case of a surgical revision. Our classification proposal is based on 3 main classes, which allow the surgeon to understand the relationships between the UP and the lacrimal bone, while the suffixes use gives additional information on the preoperative anatomy of the anterior ethmoid bone and nasal septum.

In the hope that our classification will be useful to other colleagues, we are open to further revisions and advices.

\section{Acknowledgments}

None.

\section{Conflicts of interest}

Author declares there are no conflicts of interest.

\section{Funding}

None.

\section{References}

1. Toti A. Nuovo metodo conservatore di cura radicale delle suppurazioni croniche del sacco lacrimale. Clin Med. 1904;10:385-389.

2. Dupuy-Dutemp L, Bouguet M. Note preliminaire sur un procede de dacryocystorhinostomie. Ann Oculist. 2001;158:241.

3. Tarbet KJ, Custer PL. External dacryocystorhinostomy. Surgical success, patient satisfaction and economic cost. Ophthalmology. 1995;102(7):1065-1070.

4. Warren JF, Stuart R, Seiff SR, et al. Long-Term result of external dacryocystorhinostomy. Ophthalmic Surg Lasers Imaging. 2005;36(6):446-450.

5. Caldwell GW. Two new operation for obstruction of nasal duct, with preservation of canaliculi, and an incidental description of a new lacrimal probe. $N$ York $M J .1983 ; 57: 581$

6. West JM. A window resection of the nasal duct in cases of stenosis Trans Am Ophthalmol Soc. 1910;12(pt 2):654-648

7. Mosher HP. Mosher-Toti operation on lacrimal sac. Laryngoscope 1921;31(5):284-286.

8. Sprekelsen MB, Barberan MT. Endoscopic dacryocystorhinostomy: Surgical technique and result. Laryngoscope. 1996;106(2 pt 1):187-189.

9. WoogJJ,KennedyRH,CusterPL, etal.Endonasaldacryocystorhinostomy A report by the American Academy of Ophthalmology. Ophthalmology. 2001;108(12):2369-2377.

10. Wormald PJ. Powered endonasal dacryocystorhinostomy. Laryngoscope. 2002;112(1):69-72.

11. McDonogh M, Meiring JH. Endoscopic transnasal dacryocystorhinostomy. J Laryngol Otol. 1989;103(6):585-587.

12. Massegur H, Trias E, Adema JM. Endoscopic dacryocystorhinostomy: Modified technique. Otolaryngology - Head and Neck Surgery. 2004;130(1):39-46.

13. Umapathy N, Karla S, Skinner DW,et al. Long-term results of endoscopic laser dacryocystorhinostomy. Otolaryngology - Head and Neck Surgery. 2006;135(1):81-84. 
14. Piédrola Marto D, Franco Sanchez J, Reyes Eldblom R, et al. Endonasal versus trans-canalicular endoscopic dacryocystorhinostomy using diode laser. Surgical techniques and outcome. Acta Otirrinolaringol Esp. 2008;59(6):283-287.

15. Smirnov G, Tuomilehto $H$, Terasvirta $M$, et al. Silicone tubing is not necessary after primary endoscopc dacryocystorhinostomy: a prospective randomized study. Am J Rhinol. 2008;22(2):214-217.

16. Dolmetsch AM, Gallon MA, Holds JB. Nolaser endoscopic endonasal dacryocystorhinostomy with adjunctive mitomycin $\mathrm{C}$ in children. Ophthal Plast Reconstr Surg. 2008;24(5):390-393.
17. Fayet B, Racy E, Assoline M, et al. Surgical anatomy of the lacrimal fossa a prospective computed tomodensitometry scan analysis. Ophthalmology. 2005;112(6):1119-1128.

18. Klinger F, Caviggioli F, Lisa AV, et al. Therapeutic effect of hyluronic acid reducing nasal mucosa recovery time after septoplasty. Ear Nose Throat J. 2017;96(4-5):E16-E20. 